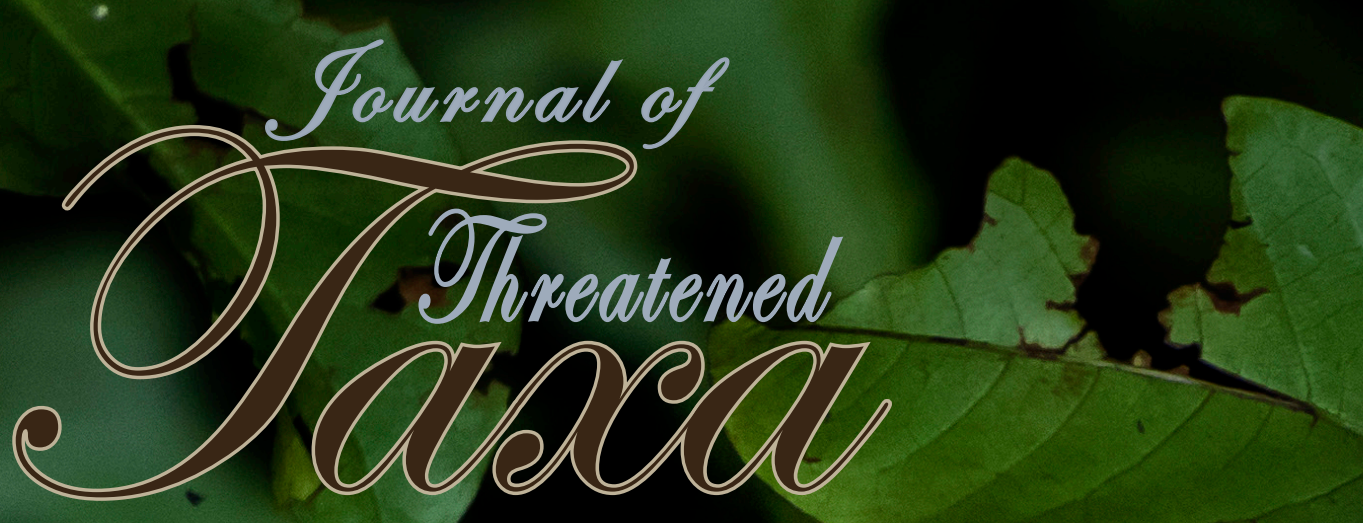

Building exidence for conservation glabally

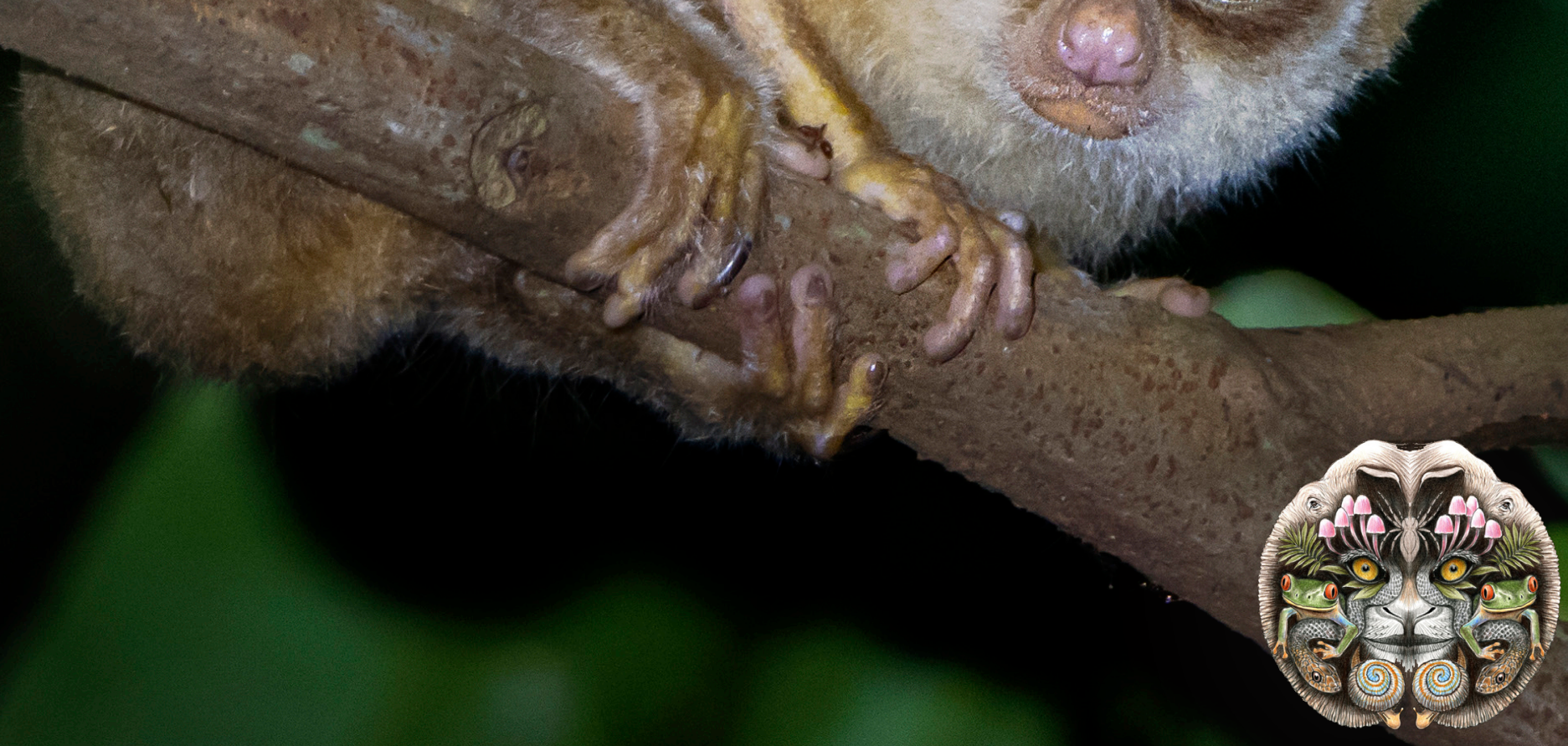

Open Access

$10.11609 /$ jott.2021.13.010.19431-19614 creven.threatenedtaxa.arg

26 September 2021 (Online \& Print) Val. 13 | Na. 11 | Pages: 19431-19674 


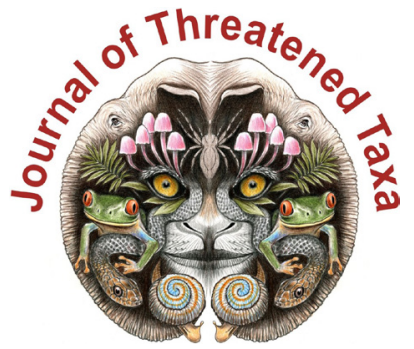

ISSN 0974-7907 (Online); ISSN $0974-7893$ (Print)

Publisher

Host

Wildlife Information Liaison Development Society

www.wild.zooreach.org

Zoo Outreach Organization www.zooreach.org

No. 12, Thiruvannamalai Nagar, Saravanampatti - Kalapatti Road, Saravanampatti, Coimbatore, Tamil Nadu 641035, India

Ph: +91 9385339863 | www.threatenedtaxa.org

Email: sanjay@threatenedtaxa.org

EDITORS

\section{Founder \& Chief Editor}

Dr. Sanjay Molur

Wildlife Information Liaison Development (WILD) Society \& Zoo Outreach Organization (ZOO)

12 Thiruvannamalai Nagar, Saravanampatti, Coimbatore, Tamil Nadu 641035, India

\section{Deputy Chief Edito}

Dr. Neelesh Dahanukar

Noida, Uttar Pradesh, India

\section{Managing Editor}

Mr. B. Ravichandran, WILD/ZOO, Coimbatore, India

\section{Associate Editors}

Dr. Mandar Paingankar, Government Science College Gadchiroli, Maharashtra 442605, India

Dr. Ulrike Streicher, Wildlife Veterinarian, Eugene, Oregon, USA

Ms. Priyanka Iyer, ZOO/WILD, Coimbatore, Tamil Nadu 641035, India

Dr. B. A. Daniel, $200 / \mathrm{WILD}$, Coimbatore, Tamil Nadu 641035, India

\section{Editorial Board}

Dr. Russel Mittermeier

Executive Vice Chair, Conservation International, Arlington, Virginia 22202, USA

\section{Prof. Mewa Singh Ph.D., FASc, FNA, FNASc, FNAPsy}

Ramanna Fellow and Life-Long Distinguished Professor, Biopsychology Laboratory, and Institute of Excellence, University of Mysore, Mysuru, Karnataka 570006, India; Honorary Professor, Jawaharlal Nehru Centre for Advanced Scientific Research, Bangalore; and Adjunct Professor, National Institute of Advanced Studies, Bangalore

\section{Stephen D. Nash}

Scientific Illustrator, Conservation International, Dept. of Anatomical Sciences, Health Sciences Center, T-8, Room 045, Stony Brook University, Stony Brook, NY 11794-8081, USA

\section{Dr. Fred Pluthero}

Toronto, Canada

\section{Dr. Priya Davidar}

Sigur Nature Trust, Chadapatti, Mavinhalla PO, Nilgiris, Tamil Nadu 643223, India

\section{Dr. Martin Fisher}

Senior Associate Professor, Battcock Centre for Experimental Astrophysics, Cavendish

Laboratory, JJ Thomson Avenue, Cambridge CB3 OHE, UK

\section{Dr. John Fellowe}

Honorary Assistant Professor, The Kadoorie Institute, 8/F, T.T. Tsui Building, The University of Hong Kong, Pokfulam Road, Hong Kong

\section{Prof. Dr. Mirco Solé}

Universidade Estadual de Santa Cruz, Departamento de Ciências Biológicas, Vice-coordenado do Programa de Pós-Graduação em Zoologia, Rodovia Ilhéus/Itabuna, Km 16 (45662-000)

Salobrinho, Ilhéus - Bahia - Brasil

\section{Dr. Rajeev Raghavan}

Professor of Taxonomy, Kerala University of Fisheries \& Ocean Studies, Kochi, Kerala, India

\section{English Editors}

Mrs. Mira Bhojwani, Pune, India

Dr. Fred Pluthero, Toronto, Canad

Mr. P. Ilangovan, Chennai, India

Web Maintenance

Mrs. Latha G. Ravikumar, ZOO/WILD, Coimbatore, India

\section{Typesetting}

Mr. Arul Jagadish, ZOO, Coimbatore, India

Mrs. Radhika, ZOO, Coimbatore, India

Mrs. Geetha, ZOO, Coimbatore India

\section{Fundraising/Communications}

Mrs. Payal B. Molur, Coimbatore, India

Subject Editors 2018-2020

Fungi

Dr. B. Shivaraju, Bengaluru, Karnataka, India

Dr. R.K. Verma, Tropical Forest Research Institute, Jabalpur, India

Dr. Vatsavaya S. Raju, Kakatiay University, Warangal, Andhra Pradesh, India

Dr. M. Krishnappa, Jnana Sahyadri, Kuvempu University, Shimoga, Karnataka, India

Dr. K.R. Sridhar, Mangalore University, Mangalagangotri, Mangalore, Karnataka, India

Dr. Gunjan Biswas, Vidyasagar University, Midnapore, West Bengal, India

Plants

Dr. G.P. Sinha, Botanical Survey of India, Allahabad, India

Dr. N.P. Balakrishnan, Ret. Joint Director, BSI, Coimbatore, India

Dr. Shonil Bhagwat, Open University and University of Oxford, UK

Prof. D.J. Bhat, Retd. Professor, Goa University, Goa, India

Dr. Ferdinando Boero, Università del Salento, Lecce, Italy

Dr. Dale R. Calder, Royal Ontaro Museum, Toronto, Ontario, Canada

Dr. Cleofas Cervancia, Univ. of Philippines Los Baños College Laguna, Philippines

Dr. F.B. Vincent Florens, University of Mauritius, Mauritius

Dr. Merlin Franco, Curtin University, Malaysia

Dr. V. Irudayaraj, St. Xavier's College, Palayamkottai, Tamil Nadu, India

Dr. B.S. Kholia, Botanical Survey of India, Gangtok, Sikkim, India

Dr. Pankaj Kumar, Kadoorie Farm and Botanic Garden Corporation, Hong Kong S.A.R., China

Dr. V. Sampath Kumar, Botanical Survey of India, Howrah, West Bengal, India

Dr. A.J. Solomon Raju, Andhra University, Visakhapatnam, India

Dr. Vijayasankar Raman, University of Mississippi, USA

Dr. B. Ravi Prasad Rao, Sri Krishnadevaraya University, Anantpur, India

Dr. K. Ravikumar, FRLHT, Bengaluru, Karnataka, India

Dr. Aparna Watve, Pune, Maharashtra, India

Dr. Qiang Liu, Xishuangbanna Tropical Botanical Garden, Yunnan, China

Dr. Noor Azhar Mohamed Shazili, Universiti Malaysia Terengganu, Kuala Terengganu, Malaysia Dr. M.K. Vasudeva Rao, Shiv Ranjani Housing Society, Pune, Maharashtra, India

Prof. A.J. Solomon Raju, Andhra University, Visakhapatnam, India

Dr. Mandar Datar, Agharkar Research Institute, Pune, Maharashtra, India

Dr. M.K. Janarthanam, Goa University, Goa, India

Dr. K. Karthigeyan, Botanical Survey of India, India

Dr. Errol Vela, University of Montpellier, Montpellier, France

Dr. P. Lakshminarasimhan, Botanical Survey of India, Howrah, India

Dr. Larry R. Noblick, Montgomery Botanical Center, Miami, USA

Dr. K. Haridasan, Pallavur, Palakkad District, Kerala, India

Dr. Analinda Manila-Fajard, University of the Philippines Los Banos, Laguna, Philippines

Dr. P.A. Sinu, Central University of Kerala, Kasaragod, Kerala, India

Dr. Afroz Alam, Banasthali Vidyapith (accredited A grade by NAAC), Rajasthan, India

Dr. K.P. Rajesh, Zamorin's Guruvayurappan College, GA College PO, Kozhikode, Kerala, India

Dr. David E. Boufford, Harvard University Herbaria, Cambridge, MA 02138-2020, USA

Dr. Ritesh Kumar Choudhary, Agharkar Research Institute, Pune, Maharashtra, India

Dr. Navendu Page, Wildlife Institute of India, Chandrabani, Dehradun, Uttarakhand, India

Invertebrates

Dr. R.K. Avasthi, Rohtak University, Haryana, India

Dr. D.B. Bastawade, Maharashtra, India

Dr. Partha Pratim Bhattacharjee, Tripura University, Suryamaninagar, India

Dr. Kailash Chandra, Zoological Survey of India, Jabalpur, Madhya Pradesh, India

Dr. Ansie Dippenaar-Schoeman, University of Pretoria, Queenswood, South Africa

Dr. Rory Dow, National Museum of natural History Naturalis, The Netherlands

Dr. Brian Fisher, California Academy of Sciences, USA

Dr. Richard Gallon, llandudno, North Wales, LL30 1UP

Dr. Hemant V. Ghate, Modern College, Pune, India

Dr. M. Monwar Hossain, Jahangirnagar University, Dhaka, Bangladesh

Mr. Jatishwor Singh Irungbam, Biology Centre CAS, Branišovská, Czech Republic

Dr. lan J. Kitching Natural History Museum, Cromwell Road, UK

Dr. George Mathew, Kerala Forest Research Institute, Peechi, India

Dr. John Noyes, Natural History Museum, London, UK

For Focus, Scope, Aims, and Policies, visit https://threatenedtaxa.org/index.php/JoTT/aims_scope
For Article Submission Guidelines, visit https://threatenedtaxa.org/index.php/JoTT/about/submissions
For Policies against Scientific Misconduct, visit https://threatenedtaxa.org/index.php/JoTT/policies_various 


\title{
Catalogue of herpetological specimens from Meghalaya, India at the Salim Ali Centre for Ornithology and Natural History
}

\author{
S.R. Chandramouli ${ }^{1}$ (D) R.S. Naveen ${ }^{2}$ (D), S. Sureshmarimuthu ${ }^{3}$ (D) S. Babu ${ }^{4}$ (D), P.V. Karunakaran ${ }^{5}$ (D) $\&$ \\ Honnavalli N. Kumara ${ }^{6}$ (D)

\footnotetext{
${ }^{1}$ Department of Ecology and Environmental Sciences, School of Life Sciences, Pondicherry University, Puducherry 605014, India.

${ }^{2-6}$ Sálim Ali Centre for Ornithology and Natural History, Anaikatty, Coimbatore, Tamil Nadu 641108, India.

${ }^{1}$ findthesnakeman@gmail.com (corresponding author), ${ }^{2}$ naveen89240@gmail.com, ${ }^{3}$ mailme.sureshmarimuthu@gmail.com,

${ }^{4}$ sanbabs@gmail.com (corresponding author), ${ }^{5}$ karunakaran.pv@gmail.com, ${ }^{6}$ honnavallik@gmail.com
}

\begin{abstract}
We present a catalogue of herpetological specimens collected from select community reserves of Meghalaya, northeastern India. The collection comprises a total of 75 species of the herpetofauna, including 29 species of amphibians from 20 genera in seven families and 46 species of reptiles from 30 genera, in 10 families. We provide the details on number of examples, sex, museum numbers, and collection details including location and collector along with the relevant remarks where applicable. A total of five species of amphibians and four species of reptiles remain to be resolved systematically since no precise name could be attributed to them.
\end{abstract}

Keywords: Amphibia, Coimbatore, community reserves, museum collection, northeastern India, Reptilia, voucher specimens

The importance of natural history collections in enriching our knowledge on various aspects of organisms such as taxonomic, morphological, ontogenetic, genetic, phylogenetic, ecological, and biogeographic facets have been highlighted since the past (Lane 1996). Apart from serving as the basis for taxonomic entities, such collections of specimens serve as an important repository of historic information on species distribution patterns as well (Shaffer et al. 1998; Rocha et al. 2014; Turney et al. 2015; Yeates et al. 2016; Da Silva et al. 2017; Hill 2017; Ceríaco et al. 2019). Most of the herpetofaunal type collections within India are deposited in two museums, namely, the Zoological Survey of India (ZSI, Kolkata), and the Bombay Natural History Society (BNHS, Mumbai). Although the collections in such major museums have been catalogued at some point (Das \& Chaturvedi 1998; Das et al. 1998; Chanda et al. 2000), there are several other institutions that house a sizable collection of specimens that often remain understudied. One such collection is in the Sálim Ali Centre for Ornithology and Natural History (SACON), Coimbatore, India. SACON is an institution under the Ministry of Environment, Forests and Climate Change, Government of India. A part of the herpetological collections at SACON from peninsular India has recently been catalogued (Ganesh et al. 2020). As a part of an ongoing study in select community reserves of Meghalaya, herpetofaunal specimens were collected by P. Karthik (research fellow of the project

Citation: Chandramouli, S.R., R.S. Naveen, S. Sureshmarimuthu, S. Babu, P.V. Karunakaran \& H.N. Kumara (2021). Catalogue of herpetological specimens from Meghalaya, India at the Salim Ali Centre for Ornithology and Natural History. Journal of Threatened Taxa 13(11): 19603-19610. https://doi.org/10.11609/ jott.7318.13.11.19603-19610

Copyright: (C) Chandramouli et al. 2021. Creative Commons Attribution 4.0 International License. JoTT allows unrestricted use, reproduction, and distribution of this article in any medium by providing adequate credit to the author(s) and the source of publication.

Funding: National Mission on Himalayan Studies (GBPNI/NMHS-2017-18/MG 32, dated: 28.03.2018).

Competing interests: The authors declare no competing interests.

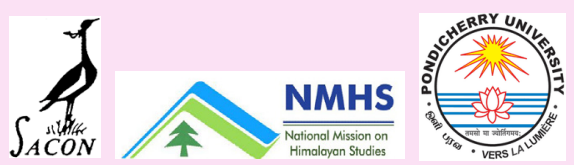

Acknowledgements: This publication is an offshoot of the project entitled 'Characterization of Community Reserves and Assessment of their Conservation Values in Meghalaya' funded by the National Mission on Himalayan Studies (GBPNI/NMHS-2017-18/MG 32, dated: 28.03.2018). Our sincere thanks are due to the additional principal chief conservator of forests (wildlife) and chief wildlife warden (CWLW), Meghalaya, and officers of the Forest and Environment Department, Government of Meghalaya for facilitating permission from the Community Reserve Management Committees to carry out the field study. We thank Mr. P. Karthik for conducting field work and collecting herpetological specimens from community reserves of Meghalaya. We are indebted to the management committees of each community reserve who gave us permission for this work. 
entitled 'Characterization of Community Reserves and Assessment of their Conservation Values in Meghalaya' funded by the National Mission on Himalayan Studies) and RSN and are deposited at SACON as vouchers. Herein, we present a catalogue of those herpetological specimens collected from Meghalaya that are maintained at SACON.

\section{MATERIALS AND METHOdS}

The herpetological specimens collected between the period 2018 to 2021 as a part of the ongoing surveys in community reserves of Meghalaya were preserved in ethanol and deposited in the collection of the SACON. Here, we list the collected specimens (only whole body, only non-larval) along with their voucher collection numbers. Institutional acronyms follow that of Ganesh et al (2020). Higher classification of amphibians and reptiles follow Frost (2021) and Uetz et al. (2021), respectively. Authorities are not mentioned for species with tentative identities indicated by 'cf.' Exceptional cases of more than one specimens catalogued under the same voucher number are distinguished by adding to their collection number the alphabets $a, b$, c etc. In cases where the specimens could not be identified precisely to the species level, the generic name only is mentioned followed by sp.

Relevant discussions based on recently published information is presented under such species to clarify their identification. Details of the collection localities mentioned below are presented in Table 1.

Catalogue of the herpetofaunal specimens from Meghalaya deposited at SACON

\section{Amphibia Gray, 1825}

Gymnophiona Müller, 1832

Ichthyophiidae Taylor, 1968

1. Ichthyophis garoensis Pillai \& Ravichandran, $1999(n=3)$

SACON VA 79 and VA 87 - two unsexed adult specimens from Dumitdigre and Sasatgre respectively (coll: P. Karthik), VA 169 - an unsexed adult from Dharibokgre (coll. R.S. Naveen).

Remark: Another putative species, Ichthyophis hussaini Pillai \& Ravichandran, 1999 from Garo Hills, Meghalaya was synonymized with I. garoensis by Kamei \& Biju (2016).

\section{Anura Fischer von Waldheim, 1813 Bufonidae Gray, 1825}

2. Duttaphrynus melanostictus (Schneider, 1799) $(n=3)$

SACON VA 55 - one adult female and VA 56 and VA 66 , two unsexed individuals, of which the former is a subadult, collected from Mongalgre (coll. P. Karthik).

3. Duttaphrynus sp. $(n=4)$

SACON VA $103 \mathrm{a}, \mathrm{b}$ - two unsexed subadults, and VA 123 and VA 124 - two adult females, from Jirang (coll. P. Karthik).

Remark: The identity of these specimens still needs resolution. Agarwal \& Mistry (2008) reported D. stuarti (Smith, 1929) from Arunachal Pradesh, and Das et al. (2013) described D. chandai from the Nagaland-Manipur border.

\section{Microhylidae Günther, 1858}

4. Microhyla berdmorei (Blyth, 1856) $(n=1)$

SACON VA 102, an adult female from Meghalaya (precise location unknown) (coll. P. Karthik).

5. Microhyla cf. mymensinghensis $(n=4)$

SACON VA 81 a, b, c - three adult females from Dumitdigre (coll. P. Karthik). VA 155 - an unsexed adult from Chimanpara (coll. R.S. Naveen).

Remark: A species described recently from the $M$. ornata complex (Hasan et al. 2014). The precise identity of these samples requires further study.

\section{Megophryidae Bonaparte, 1850}

6. Leptobrachium cf. sylheticum $(n=6)$

SACON VA 57, VA 61 - two adult females from Mongalgre; VA 58, VA 59, VA 60 - three unsexed adult specimens and VA 151 - an unsexed adult from Eman Asakgre (coll. R.S. Naveen).

Remarks: The reports of another species, $L$. rakhinense Wogan, 2012, from Northeast India have been shown by Dutta et al (2013) to represent L. smithi. Very recently, populations of the 'L.smithi' complex were reassessed by Al-Razi et al (2021) and described as a new species. Considering the geographic proximity of our samples to the type locality of $L$. sylheticum, we refer our specimens as $L$. cf. sylheticum.

7. Leptobrachella cf. khasiorum $(n=1)$

VA 115 - an unsexed subadult from Jirang (coll. P. Karthik)

8. Xenophrys major (Boulenger, 1908) $(n=1)$

SACON VA 83 - an adult female from Mongalgre (coll. P. Karthik).

Remark: The genus Xenophrys Günther, 1864, which was placed under the synonymy of Megophrys Kuhl \& Van Hasselt, 1822 by Mahony et al. (2013) has now been revalidated by Lyu et al (2021). 
Table 1. GPS coordinates of collection localities in Meghalaya, India.

\begin{tabular}{|c|c|c|c|c|c|}
\hline & $\begin{array}{l}\text { Community } \\
\text { Reserves / Sites }\end{array}$ & District & $\begin{array}{c}\text { Latitude } \\
\left({ }^{\circ} \mathrm{N}\right)\end{array}$ & $\begin{array}{c}\text { Longitude } \\
\left({ }^{\circ} \mathrm{E}\right)\end{array}$ & $\begin{array}{l}\text { Altitude } \\
\text { (m) }\end{array}$ \\
\hline 1 & Chandigre & West Garo Hills & 25.5362 & 90.3256 & 833 \\
\hline 2 & Dalu & West Garo Hills & 25.2206 & 90.2163 & 31 \\
\hline 3 & Daribokgre & North Garo Hills & 25.47902 & 90.3105 & 1123 \\
\hline 4 & Mongalgre & West Garo Hills & 25.6261 & 90.2064 & 535 \\
\hline 5 & Sakalgre & West Garo Hills & 25.5143 & 90.3808 & 895 \\
\hline 6 & Sasatgre & West Garo Hills & 25.5262 & 90.3283 & 895 \\
\hline 7 & Selbalgre & West Garo Hills & 25.5143 & 90.2030 & 282 \\
\hline 8 & Tura & West Garo Hills & 25.515 & 90.2027 & 281 \\
\hline 9 & Kitmadamgre & North Garo Hills & 25.8006 & 90.3959 & 223 \\
\hline 10 & Eman Asakgre & South Garo Hills & 25.36989 & 90.54481 & 174 \\
\hline 11 & Thokpara & West Garo Hills & 25.2756 & 90.1051 & 94 \\
\hline 12 & Dangkipara & South Garo Hills & 25.4286 & 90.3269 & 380 \\
\hline 13 & Chimanpara & West Garo Hills & 25.29606 & 90.12145 & 92 \\
\hline 14 & Rongalgre & West Garo Hills & 25.4574 & 90.1669 & 112 \\
\hline 15 & Dumitdigre & West Garo Hills & 25.6084 & 92.0156 & 1103 \\
\hline 16 & NEHU, Shillong & East Khasi Hills & 25.6126 & 91.8972 & 1404 \\
\hline 17 & Jirang & Ri Bhoi & 25.8974 & 91.5849 & 647 \\
\hline 18 & Lum Jusong & Ri Bhoi & 25.8948 & 92.0396 & 919 \\
\hline 19 & Nongpoh & Ri Bhoi & 25.8983 & 91.8956 & 681 \\
\hline 20 & Nongsangu & Ri Bhoi & 25.8717 & 92.0529 & 740 \\
\hline 21 & Raid Nongbri & Ri Bhoi & 25.9152 & 92.0156 & 790 \\
\hline
\end{tabular}

NEHU-North Eastern Hill University

9. Xenophrys megacephala (Mahony, Sengupta, Kamei \& Biju, 2011) ( $n=1)$

VA 80 - an unsexed adult specimen from Dumitdigre (coll. P. Karthik).

Remark: See above for taxonomic validity of the genus Xenophrys Günther, 1864.

10. Xenophrys oropedion (Mahony, Teeling \& Biju, 2013) $(n=1)$

VA 67 - an adult female from Daribokgre (coll. P. Karthik)

Remark: See above for taxonomic validity of the genus Xenophrys Günther, 1864.

11. Xenophrys sp. $(n=1)$

VA 86 - an unsexed subadult from Sasatgre (coll. P. Karthik), whose identity could not be determined.

\section{Dicroglossidae Anderson, 1871}

12. Fejervarya sp. $(n=5)$

VA 54, VA 82 and VA 98 - three adult females from Mongalgre and Lum Jusong, respectively. VA 75 - an adult male from Dumitdigre. VA 107 - an unsexed subadult from Lum Jusong (coll. P. Karthik).

Remark: A large-bodied Fejervarya frog, F. orissaensis
Dutta, 1997 has recently been shown to occur across most parts of Indochina (Köhler et al. 2019). The identity of our Fejervarya specimens still needs taxonomic resolution.

13. Minervarya sengupti (Purkayastha \& Matsui, 2012) $(n=10)$

Ten specimens. VA 117-119 - three adult females from Jirang. VA 62 - one adult female. VA 63-65 - three adult males from Daribokgre. VA 71 - one adult female from Dumitdigre. VA 89, VA 97 - two adult females from Meghalaya (precise location unavailable) (coll. P. Karthik).

Remark: A fairly recently described species from Mawphlang, Khasi Hills, Meghalaya (Purkayastha \& Matsui 2012).

14. Minervarya cf. pierrei $(n=7)$

VA 72, VA 73 and VA 74 - three adult males from Dumitdigre. VA 116 - an adult female from Jirang. VA 92 an adult female from Daribokgre and VA 84-85 - two unsexed adult specimens from Sasatgre (coll. P. Karthik).

Remark: The taxonomic status and distribution of Minervarya pierrei (Dubois, 1975) and Minervarya agricola (Jerdon, 1853) were recently discussed by 
Chandramouli et al. (2019) and Phuge et al. (2020).

15. Minervarya sp. $(n=1)$

VA 109 - an unsexed juvenile specimen from Meghalaya (coll. P. Karthik) that could not be identified to species level.

16. Limnonectes khasianus (Anderson, 1871) $(n=$ 8)

VA 111, VA 112 - two adult males from Jirang, VA 99 and VA 69 - two adult males from Dimitdigre, VA 68 - an unsexed adult from Meghalaya (precise locality unknown) (coll. P. Karthik), VA 130-131, two unsexed adults from Rongalgre and VA 132, an unsexed subadult from Kitmadamgre (coll. R.S. Naveen).

Remark: Ohler \& Deuti (2013) discussed and confirmed the synonymy of Rana laticeps Boulenger, 1882 with Pyxicephalus khasianus Anderson, 1871, thereby highlighting the seniority of the name combination Limnonectes khasianus (Anderson, 1871). 4)

17. Euphlyctis cyanophlyctis (Schneider, 1799) $(\mathrm{n}=$

VA 94 - one adult female (coll. P. Karthik). VA 113, VA 114, VA 125 - three unsexed subadults from Jirang (coll. P. Karthik).

18. Ingerana borealis (Annandale, 1912) $(n=5)$

VA 135-138, four unsexed adults from Rongalgre, VA 161 - an adult female from Rongalgre (coll. R.S. Naveen).

\section{Ranidae Batsch, 1796}

19. Clinotarsus alticola (Boulenger, 1882) $(n=4)$

VA 95 and VA 106 - two adult females, VA 110 - a juvenile and VA 91 - a subadult from Sasatgre (coll. P. Karthik).

Remarks: Members of the genus Clinotarsus Minvart, 1869 show a disjunct pattern of geographic distribution. While C. curtipes (Jerdon, 1853) is restricted to the Western Ghats of southwestern peninsular India, the other two congeners C. alticola (Boulenger, 1882) and C. penelope Grosjean, Bordoloi, Chuaynkern, Chakravarty \& Ohler, 2015 occur in the Indochinese region.

20. Hylarana tytleri Theobald, $1868(n=1)$

VA 93 - one unsexed subadult from Lum Jusong (coll. P. Karthik).

21. Hydrophylax leptoglossa (Cope, 1868) $(n=2)$

VA 100-101 - two adult females from Sasatgre (coll. P. Karthik).

22. Amolops assamensis Sengupta, Hussain, Choudhury, Gogoi, Ahmed \& Choudhury, 2008 ( $n=1)$

VA 52 - an unsexed subadult from Jirang,(coll. P. Karthik).

23. Amolops marmoratus (Blyth, 1855) $(n=2)$

VA 90a-b - two unsexed juveniles from Sasatgre (coll.
P. Karthik).

24. Amolops sp. $(n=3)$

VA 120-122 - three subadult females from Jirang. Their identity could not be determined to species level.

\section{Rhacophoridae Hoffman, 1932}

25. Polypedates himalayensis (Annandale, 1912) $(n=3)$

VA 76, VA 77 and VA 78 - Three adult females, from Dumitdigre (coll. P. Karthik).

26. Polypedates $\mathrm{cf}$. leucomystax $(\mathrm{n}=1)$

VA 162, an unsexed adult from Tura (coll. R.S. Naveen).

Remark: The identity of $P$. leucomystax from India still needs finer taxonomic resolution (Frost 2021).

27. Raorchestes sp. $(n=9)$

VA 51 a\&b, VA 105 - respectively, two adult males and an unsexed adult specimen from Mongalgre (coll. P. Karthik), VA 126-128, three unsexed adults from Sakalgre and VA 129 one from Daribokgre, VA 149-150 - two adult males from Sasatgre and Eman Asakgre respectively (coll. R.S. Naveen).

Remarks: Boruah et al (2018) presented point localities for $R$. shillongensis Pillai \& Chanda, 1973 from Khasi Hills, lying to nearly $20 \mathrm{~km}$ to the east of Mongalgre. The identity of the specimens recorded here requires further study.

28. Theloderma baibungense (Jiang, Fei \& Huang, 2009) $(n=2)$

VA 88, VA 96 - an unsexed juvenile and an adult female from Selbalgre and Raid Nongbri respectively (coll. P. Karthik).

29. Kurixalus naso (Annandale, 1912) $(n=2)$

VA 134, VA153 unsexed adults from Eman Asakgre and Sasatgre respectively (coll. R.S. Naveen).

Remark: Lalronunga et al. (2021) presented records of $K$. yangi from Mizoram and discussed their distribution records and confusions on the identities of the two species, indicating a possible synonymy of $K$. yangi with K. naso.

Reptilia Laurenti, 1768

Sauria Macartney, 1802

Gekkonidae Gray, 1825

30. Cnemaspis assamensis Das \& Sengupta, 2000 $(n=3)$

VR 237, VR 233 and VR 221 - Three adults; one male, one female and an unsexed from Raid Nongbri, respectively (coll. P. Karthik).

31. Cyrtodactylus cf. agarwali $(n=6)$ 
VR 230-231, two adult males, from Sasatgre; VR 181-183 three adults from Daribokgre; and VR 153- one juvenile from Mongalgre (coll. P. Karthik).

Remark: Purkayasta et al. (2020) recently reported another species, C. urbanus Purkayastha, Das, Bohra, Bauer \& Agarwal, 2020 from Nongpoh. Additionally, Purkayasta et al. (2021) described two more new species C. agarwali and C. karsticola from the Garo Hills.

32. Hemidactylus platyurus (Schneider, 1797) ( $n=$ 7)

VR 198, VR 218a and VR 232 - three adult males and VR $218 b$ - one adult female from Mongalgre, VR 195, VR 200 and VR 216 three adult females from Sasatgre (coll. P. Karthik).

33. Hemidactylus frenatus Duméril \& Bibron, 1836 $(n=1)$

VR 222 - subadult from Meghalaya (no more precise locality) (coll. P. Karthik).

34. Hemidactylus sp. $(n=1)$

VR 171 - subadult male from Meghalaya (no more precise locality) (coll. P. Karthik).

35. Gekko gecko (Linnaeus, 1758) $(n=1)$

VR 229 - adult male from Meghalaya (no more precise location) (coll. P. Karthik).

\section{Agamidae Gray, 1827}

36. Calotes cf. irawadi $(n=9)$

VR 178 a \& b - an unsexed and an adult female from Sasatgre; VR 205, VR 240-245- six unsexed subadult specimens respectively from Meghalaya (no more precise location) (coll. P. Karthik).

Remarks: Zug et al. (2006) described Calotes irawadi from Myanmar. The exact identity of our samples from Meghalaya still needs further investigation regarding their potential conspecificity with that newly described taxon.

37. Calotes maria Gray, $1845(n=2)$

VR 166, 173 - two adults respectively from Daribokgre and Sasatgre (coll. P. Karthik).

38. Calotes emma Gray, $1845(n=3)$

VR 247, VR 150, VR 151 - one adult from Dumitdigre, two adults respectively from Meghalaya (no more precise location) (coll. P. Karthik).

39. Calotes sp. $(n=2)$

VR 206, 251 - respectively, an unsexed subadult and adult male from Dumitdigre (coll. P. Karthik).

Remark: Species is uncertain and needs to be determined.

40. Cristidorsa planidorsata (Jerdon, 1870) $(n=4)$

VR 185 and VR 169 - two adult males from Meghalaya (no more precise location); VR 184 and VR 188- one adult female each from Daribokgre and Sasatgre, respectively (coll. P. Karthik).

41. Ptyctolaemus gularis (Peter, 1864) $(n=8)$

VR 238, VR 239, VR 207 - three adult males and, VR 201 - an unsexed juvenile from Meghalaya (no more precise location), VR 167, VR 168, VR 179 and VR 180 - four unsexed adults from Daribokgre (coll. P. Karthik).

Scincidae Gray, 1825

42. Sphenomorphus indicus (Gray, 1853) $(n=3)$

VR 186, VR 224, VR 249 - three unsexed adults respectively from Daribokgre, Sasatgre, and Dumitdigre (coll. P. Karthik).

43. Sphenomorphus maculatus (Blyth, 1853) $(n=7)$

VR 164, VR 165, VR 197, VR 234 a\&b - five unsexed adults and VR 217 and VR 226 - two subadults from Sasatgre (coll. P. Karthik).

44. Sphenomorphus sp. $(n=1)$

VR 227 - subadult from Meghalaya (no more precise location) (coll. P. Karthik).

45. Eutropis multifasciata (Kuhl, 1820) $(n=1)$

VR 169 - juvenile from Nongsangu.

46. Eutropis cf. macularia $(n=4)$

VR 199 - one juvenile, VR198 - one subadult and VR 235 and VR 236 - two adults from Lum Jusong (coll. P. Karthik).

\section{Lacertidae Oppel, 1811}

47. Takydromus khasiensis Boulenger, $1917(n=2)$

VR 155, 208 - two unsexed adults respectively from Mongalgre and Nongsangu (coll. P. Karthik).

\section{Serpentes Linnaeus, 1758 \\ Typhlopidae Merrem, 1820}

48. Argyrophis diardii (Schlegel, 1839) $(n=4)$

VR 187, 223 - two adult specimens respectively from Daribokgre and Sasatgre (coll. P. Karthik), VR 255-256 - one adult and subadult respectively from Dangkipara (coll. R.S. Naveen).

49. Indotyphlops sp. $(\mathrm{n}=1)$

An unsexed adult specimen (VA 219) from Meghalaya (no more precise location) (coll. P. Karthik).

Remark: Superficially resembles I. braminus (Daudin, 1803) but the precise identity of this specimen requires further study.

\section{Pseudaspididae Cope, 1893}

50. Psammodynastes pulverulentus (Boie, 1827) $(n=1)$

VR 152 - a subadult specimen from Meghalaya (no more precise location) (coll. P. Karthik). 
Colubridae Oppel, 1811

51. Calamaria parvimentata Duméril, Bibron \& Duméril, $1854(\mathrm{n}=1)$

VR 261 - an unsexed adult from Daribokre (coll. R.S. Naveen).

52. Lycodon zawi Slowinski, Pawar, Win, Thin, Gyi, Oo \& Tun, $2001(n=1)$

VR 204 - an unsexed adult specimen from Lum Jusong (coll. P. Karthik).

53. Lycodon sp. $(n=2)$

VR 213, VR 215 - two subadult specimens from Meghalaya (no more precise location) (coll. P. Karthik). Their specific identity needs further study.

54. Lycodon jara (Shaw, 1802) $(n=1)$

VR 253, an unsexed adult from Thokpara (coll. R.S. Naveen).

55. Lycodon cf. aulicus $(\mathrm{n}=1)$

VR 254, an unsexed adult from Thokpara (coll. R.S. Naveen).

56. Oligodon juglandifer (Wall, 1909) $(n=1)$

VR 214 - unsexed adult road killed specimen from Meghalaya (no more precise location) (coll. P. Karthik).

57. Oligodon cyclurus (Cantor, 1839) $(n=1)$

VR 254 - an unsexed adult from Thokpara.

58. Boiga cyanea (Duméril, Bibron \& Duméril, 1854) $(n=1)$

VR 228 - a large adult specimen from Nongsangu (coll. P. Karthik).

59. Boiga gocool (Gray, 1834) $(n=3)$

VR 190-192 - unsexed subadults from Meghalaya (no more precise location) (coll. P. Karthik).

60. Dendrelaphis proarchos (Wall, 1909) $(n=1)$

VR 210 - adult from Meghalaya (no more precise location) (coll. P. Karthik).

Remark: Vogel \& Van Rooijen (2011) revalidated $D$. proarchos from the synonymy of $D$. pictus which has recently been endorsed by Hakim et al. (2020).

61. Coelognathus radiatus (Boie, 1827) $(n=1)$

VR 189 - subadult from Meghalaya (no more precise location) (coll. P. Karthik).

62. Elaphe cantoris (Boulenger, 1894) $(n=1)$

VR 211 - an unsexed adult (VR 211) from Meghalaya (no more precise location) (coll. P. Karthik).

\section{Pareidae Romer, 1956}

63. Pareas monticola (Cantor, 1839) $(n=1)$

VR 212 - adult from Meghalaya (no more precise location) (coll. P. Karthik).

\section{Natricidae Bonaparte, 1838}

64. Pseudoxenodon macrops (Blyth, 1855) $(n=1)$
VR 260 - an adult male from Chandigre (coll. R.S. Naveen).

65. Trachischium monticola (Cantor, 1839) $(n=3)$

VR 163, VR 172, VR 220 - adults from Daribokgre (coll. P. Karthik).

66. Hebius khasiense (Boulenger, 1890) $(n=8)$

VR 162, VR 175-177 four unsexed adults from Sasatgre, VR 209, VR 225, VR 246 - three unsexed adults from Meghalaya (no more precise precise location) (coll. P. Karthik), VR 257 - an unsexed adult from Sasatgre (coll. R.S. Naveen).

67. Fowlea piscator (Schneider, 1799) $(n=3)$

VR 156 - adult male road killed specimen from Nongsangu. VA 202-203 - adults from Meghalaya (no more precise location) (coll. P. Karthik).

Remarks: Purkayastha et al. (2018) allocated Xenochrophis piscator to the genus Fowlea Theobald, 1868.

68. Smithophis bicolor (Blyth, 1854) $(n=1)$

VR 194 - subadult male from Northeastern Hill University Campus, Shillong (coll. P. Karthik).

Remarks: This specimen was recently described in detail by Chandramouli et al. (2021).

\section{Elapidae Boie, 1827}

69. Sinomicrurus macclellandi (Reinhardt, 1844) $(n=1)$

VR 159 - one adult from Meghalaya (no more precise location) (coll. P. Karthik).

70. Naja kaouthia Lesson, $1831(\mathrm{n}=1)$

VR 157 - one juvenile from Meghalaya (no more precise location) (coll. P. Karthik).

71. Ophiophagus hannah (Cantor, 1836) $(n=1)$

VR 252 - an adult male from Meghalaya (no more precise location) (coll. P. Karthik).

\section{Viperidae Oppel, 1811}

72. Ovophis monticola (Günther, 1864) $(n=3)$

VR 161, VR 193, VR 248 - three adults from Dumitdigre (coll. P. Karthik).

73. Trimeresurus popeiorum Smith, $1937(n=2)$

VR 170, VR 174 - two adults, respectively one male and one female from Daribokgre and Sasatgre (coll. P. Karthik).

74. Trimeresurus erythrurus (Cantor, 1839) $(n=2)$

VR 158 - subadult from Selbalgre (coll. P. Karthik), VR 259 - a subadult from Dalu (coll. R.S. Naveen).

75. Trimeresurus sp. $(n=1)$

VR 160 - one subadult, (VR 160) from Meghalaya (no more precise location) (coll. P. Karthik), whose specific identity needs further study. 


\section{Discussion}

Currently, the collection encompasses a total of 75 species of the herpetofauna, including 29 species of amphibians from 20 genera in seven families and 46 species of reptiles from 31 genera, in 10 families. Reptiles are represented by 17 species of lizards and 29 species of snakes. This collection is expected to grow as the field study continues. The collections from peninsular India at SACON have recently been catalogued (Ganesh et al. 2020) and there still are collections from other regions within India that will be cataloged in future. Herpetofaunal collections in other institutions within India are recently being catalogued (e.g., Ganesh 2010; Ganesh \& Asokan 2010; Zacharias \& Jose 2020) which would aid in supplementing our knowledge on herpetofaunal species and their distribution.

\section{REFERENCES}

Agarwal, I. \& V.K. Mistry (2008). Bufo stuarti from western Arunacha Pradesh, India. Russian Journal of Herpetology 15: 166-168.

Al-Razi, H., M. Maria \& N. Poyarkov (2021). Integrative taxonomic analysis reveals a new species of Leptobrachium Tschudi, 1838 (Anura, Megophryidae) from Bangladesh. Journal of Natural History 55(1-2): 85-114.

Boruah, B., P. Raj, S.K. Dutta \& A. Das (2018). Redescription and geographic distribution of Raorchestes shillongensis (Anura: Rhacophoridae) from Meghalaya, Northeast India. Phyllomedusa 17: 3-20.

Ceríaco, L.M.P. \& M.M. Pimentel (2019). New Uses for an Old and Abandoned Colonial Collection: The herpetological collection of the Instituto de Investigação Científica Tropical (Lisbon, Portugal). Biodiversity Information Science and Standards 3: e37268.

Chanda, S.K., I. Das \& A. Dubois (2000). Catalogue of amphibian types in the collection of the zoological survey of India. Hamadryad 25: 100-128.

Chandramouli, S.R., D. Ankaiah, V. Arul, S.K. Dutt \& S.R. Ganesh (2019). On the taxonomic status of Minervarya granosa (Kuramoto, Joshy, Kurabayashi \& Sumida, 2008) and the distribution of $M$. agricola (Jerdon, 1853) Amphibia: Anura: Dicroglossidae. Asian Journal of Conservation Biology 8: 84-87.

Chandramouli, S.R., P. Karthik, R.S. Naveen, S. Babu, P.V. Karunakaran \& H.N. Kumara (2021). A two-colored forestsnake, Smithophis bicolor (Blyth 1855) (Reptilia: Natricidae), from the Khasi Hills, Meghalaya, India. Reptiles \& Amphibians 28(1): 24-25.

Da Silva, F.R., D.B. Provete, L.K. Gerassi \& R.P. Bovo (2017). What do Data from Fieldwork and Scientific Collections Tell us about Species Richness and Composition of Amphibians and Reptiles? South American Journal of Herpetology 12: 99-106.

Das, A., M. Chetia, S.K. Dutta \& S. Sengupta (2013). A new species of Duttaphrynus (Anura: Bufonidae) from northeast India. Zootaxa 3646(4): 336-348.

Das, I. \& N. Chaturvedi (1998). Catalogue of the herpetological types in the collection of the Bombay Natural History Society. Hamadryad 23(2): 150-156.

Das, I., B. Dattagupta \& N.C. Gayen (1998). History and catalogue of reptile types in the collection of the Zoological Survey of India. Journal of South Asian Natural History 3(2): 121-172.

Dutta, D., A. Das, A. Dutta, J. Gogoi \& S. Sengupta (2013). Taxonomic status and distribution of Leptobrachium smith Matsui, Nabhitabhata \& Panha, 1999 (Anura: Megophryidae) in India with new locality records. Tropical Natural History 13: 87-95.

Frost, D.R. (2021). Amphibian Species of the World: an Online
Reference. Version 6.1 (Date of access). Electronic Database accessible at https://amphibiansoftheworld.amnh.org/index.php. American Museum of Natural History, New York, USA. https://doi. org/10.5531/db.vz.0001

Ganesh, S.R (2010). Catalogue of herpetological specimens in the Chennai Snake Park. Cobra 4: 1-22.

Ganesh, S.R. \& J.R. Asokan (2010). Catalogue of Indian herpetological specimens in the collection of the Government Museum Chennai, India. Hamadryad 35: 46-63.

Ganesh, S.R., S. Bhupathy, P. Karthik, B. Rao \& S. Babu (2020). Catalogue of herpetological specimens from peninsular India at the Sálim Ali Centre for Ornithology \& Natural History (SACON), India. Journal of Threatened Taxa 12(9): 16123-16135. https://doi. org/10.11609/jott.6036.12.9.16123-16135

Hakim, J., S.J. Trageser, A. Ghose, K. Das, S.M.A. Rashid \& S.C. Rahman (2020). Amphibians and reptiles from Lawachara National Park in Bangladesh. Check List 16 (5): 1239-1268. https://doi. org/10.15560/16.5.1239

Hasan, M.K., M.M. Islam, M. Kuramoto, A. Kurabayashi \& M. Sumida (2014). Description of two new species of Microhyla (Anura: Microhylidae) from Bangladesh. Zootaxa 3755: 401-408.

Hill, J.E. (2017). Museum specimens answer question of historic occurrence of Nile tilapia Oreochromis niloticus (Linnaeus, 1758) in Florida (USA). Biolnvasions Records 6: 383-391.

Kamei, R.G. \& Biju S.D (2016). On the taxonomic status of Ichthyophis husaini Pillai \& Ravichandran, 1999 (Amphibia: Gymnophiona: Ichthyophiidae). Zootaxa 4079(1): 140-150. https://doi. org/10.11646/zootaxa.4079.1.10

Köhler, G., L. Mogk, K.P.P. Khaing \& N.L. Than (2019). The genera Fejervarya and Minervarya in Myanmar: Description of a new species, new country records, and taxonomic notes (Amphibia, Anura, Dicroglossidae. Vertebrate Zoology 69: 183-226.

Lalronunga, S., Vanramliana, Lalramliana \& E. Lalhmingliani (2021). A new country record of Raorchestes cangyuanensis $\mathrm{Wu}$, Suwannapoom, Xu, Murphy \& Che, 2019 and additional record of Kurixalus yangi Yu, Hui, Rao \& Yang, 2018 (Anura: Rhacophoridae: Rhacophorinae) from India. Zootaxa 4974: 383-390. https://doi. org/10.11646/zootaxa.4974.2.7

Lane, M. (1996). Roles of Natural History Collections. Annals of the Missouri Botanical Garden 83(4): 536-545. https://doi. org/10.2307/2399994

Lyu, Z.-T., Z. Zeng, J. Wang, Z.-Y. Liu, Y.-Q. Huang, W.-Z. Li \& Y.-Y. Wang (2021). Four new species of Panophrys (Anura, Megophryidae) from eastern China, with discussion on the recognition of Panophrys as a distinct genus. Zootaxa 4927: 9-40. https://doi.org/10.11646/ zootaxa.4927.1.2.

Mahony, S., E.C. Teeling \& S.D. Biju (2013). Three new species of horned frogs, Megophrys (Amphibia: Megophryidae), from northeast India, with a resolution to the identity of Megophrys boettgeri populations reported from the region. Zootaxa 3722: 143-169.

Ohler, A. \& K. Deuti (2013). Pyxicephalus khasianus Anderson, 1871 and Rana laticeps Boulenger, 1882 (Dicroglossidae, Anura, Amphibia) are synonyms. Zoosystema 35(3): 415-424.

Phuge, S., A.B. Patil, R. Pandit, N.U. Kulkarni, B.H. Chennakeshavamurthy, P. Deepak \& K.P. Dinesh (2020). Importance of genetic data in resolving cryptic species: a century old problem of understanding the distribution of Minervarya syhadrensis Annandale 1919, (Anura: Dicroglossidae). Zootaxa 4869: 451-492. https://doi.org/10.11646/zootaxa.4869.4.1

Purkayastha, J. \& M. Matsui (2012). A new species of Fejervarya (Anura: Dicroglossidae) from Mawphlang, northeastern India. Asian Herpetological Research 2, 3: 31-37.

Purkayastha, J., J. Kalita, R.K. Brahma, R. Doley \& M. Das (2018). A review of the relationships of Xenochrophis cerasogaster Cantor, 1839 (Serpentes: Colubridae) to its congeners. Zootaxa 4514 (1): 126-136. https://doi.org/10.11646/zootaxa.4514.1.10

Purkayastha, J., S.C. Bohra \& M. Das (2020). First Record of the Urban Bent-toed Gecko, Cyrtodactylus urbanus Purkayastha, Das, Bohra, Bauer, and Agarwal 2018 (Squamata: Gekkonidae), from Meghalaya, 
India. Reptiles and Amphibians 27(3):512-513.

Purkayastha, J., H.T. Lalremsanga, S.C. Bohra, L. Biakzuala, H.T. Decemson, L. Muansanga, M. Vabeiryureilai, S. Chauhan \& Y.S. Rathee (2021). Four new Bent-toed geckos (Cyrtodactylus Gray: Squamata: Gekkonidae) from northeast India. Zootaxa 4980 (1): 451-489. https://doi.org/10.11646/zootaxa.4980.3.2

Rocha, L.A., A. Aleixo, G. Allen, F. Almeda, C.C. Baldwin, M.V.L. Barclay, J.M. Bates, A.M. Bauer, F., Benzoni, C.M. Berns, M.L. Berumen, D.C. Blackburn, S. Blum, F. Bolaños, R.C.K. Bowie, R. Britz, R.M. Brown, C.D. Cadena, K. Carpenter, L.M. Ceríaco, P. Chakrabarty, G. Chaves, J.H. Choat, K.D. Clements, B.B. Collette A. Collins, J. Coyne, J. Cracraft, T. Daniel, M.R. de Carvalho, K. de Queiroz, F. di Dario, R. Drewes, J.P. Dumbacher, A. Engilis JR., M.V. Erdmann, W. Eschmeyer, C.R. Feldman, B.L. Fisher, J. Fjeldså, P.W. Fritsch, J. Fuchs, A. Getahun, A. Gill, M. Gomon, T. Gosliner, G.R. Graves, C.E. Griswold, R. Guralnick, K. Hartel, K.M. Helgen, H. Ho, D.T. Iskandar, T. Iwamoto, Z. Jaafar, H.F. James, D. Johnson, D. Kavanaugh, N. Knowlton, E. Lacey, H. K. Larson, P. Last, J. M. Leis, H. Lessios, J. Liebherr, M. Lowman, D.L. Mahler, V. Mamonekene, K. Matsuura, G.C. Mayer, H. Mays Jr., J. Mccosker, R.W. Mcdiarmid, J. McGuire, M.J. Miller, R. Mooi, R.D. Mooi, C. Moritz, P. Myers, M.W. Nachman, R.A. Nussbaum, D.O. Foighil, L.R. Parenti, J.F. Parham, E. Paul, G. Paulay, J. Pérez-emán, A. Pérez-matus, S. Poe, J. Pogonoski, D.L. Rabosky, J.E. Randall, J.D. Reimer, D.R. Robertson, M.O. Rödel, M.T. Rodrigues, P. Roopnarine, L. Rüber, M.J. Ryan, F. Sheldon, G. Shinohara, A. Short, W.B. Simison, W. F. Smith-vaniz, V.G. Springer, M. Stiassny, J.G. Tello, C.W. Thompson, T. Trnski, P. Tucker, T. Valqui, M. Vecchione, E. Verheyen, P.C. Wainwright, T.A. Wheeler, W.T. White, K. Will, J.T. Williams, G. Williams, E.O. Wilson, K. Winker, R. Winterbottom \& C.C. Witt (2014). Specimen collection: An essential tool. Science 344(6186): 814-816.
Shaffer, H.B., R.N. Fisher \& C. Davidson (1998). The role of natural history collections in documenting species declines. Trends in Ecology \& Evolution 13: 27-30.

Turney, S., E.R. Cameron, C.A. Cloutier \& C.M. Buddle (2015). Nonrepeatable science: assessing the frequency of voucher specimen deposition reveals that most arthropod research cannot be verified. PeerJ 3: e1168. https://doi.org/10.7717/peerj.1168

Uetz, P., P. Freed, R. Aguilar \& J. Hošek (eds.) (2021). The Reptile Database, http://www.reptile-database.org, accessed on 04 July 2021

Vogel, G. \& J. van Rooijen (2011). Contributions to a review of the Dendrelaphis pictus (Gmelin, 1789) complex (Serpentes: Colubridae)-3. The Indian forms, with the description of a new species from the Western Ghats. Journal of Herpetology 45(1): 100-110.

Yeates, D.K., A. Zwick \& A.S. Mikheyev (2016). Museums are biobanks: unlocking the genetic potential of the three billion specimens in the world's biological collections. Current Opinion in Insect Science 18: 83-88.

Zacharias, V.J. \& B. Jose (2020). An account of snake specimens in St. Joseph's College Museum Kozhikode, India, with data on species diversity. Journal of Threatened Taxa 12(11): 16622-16627. https:// doi.org/10.11609/jott.4995.12.11.16622-16627

Zug, G.R., H.H.K. Brown, J.A. Schulte II \& J.V. Vindum (2006). Systematics of the Garden Lizards, Calotes versicolor Group (Reptilia, Squamata, Agamidae), in Myanmar: Central Dry Zone Populations. Proceedings of the Caifornia Academy of Sciences 57(2): 35-68. 
Dr. Albert G. Orr, Griffith University, Nathan, Australia

Dr. Sameer Padhye, Katholieke Universiteit Leuven, Belgium

Dr. Nancy van der Poorten, Toronto, Canada

Dr. Kareen Schnabel, NIWA, Wellington, New Zealand

Dr. R.M. Sharma, (Retd.) Scientist, Zoological Survey of India, Pune, India

Dr. Manju Siliwal, WILD, Coimbatore, Tamil Nadu, India

Dr. G.P. Sinha, Botanical Survey of India, Allahabad, India

Dr. K.A. Subramanian, Zoological Survey of India, New Alipore, Kolkata, India

Dr. P.M. Sureshan, Zoological Survey of India, Kozhikode, Kerala, India

Dr. R. Varatharajan, Manipur University, Imphal, Manipur, India

Dr. Eduard Vives, Museu de Ciències Naturals de Barcelona, Terrassa, Spain

Dr. James Young, Hong Kong Lepidopterists' Society, Hong Kong

Dr. R. Sundararaj, Institute of Wood Science \& Technology, Bengaluru, India

Dr. M. Nithyanandan, Environmental Department, La Ala Al Kuwait Real Estate. Co. K.S.C., Kuwait

Dr. Himender Bharti, Punjabi University, Punjab, India

Mr. Purnendu Roy, London, UK

Dr. Saito Motoki, The Butterfly Society of Japan, Tokyo, Japan

Dr. Sanjay Sondhi, TITLI TRUST, Kalpavriksh, Dehradun, India

Dr. Nguyen Thi Phuong Lien, Vietnam Academy of Science and Technology, Hanoi, Vietnam

Dr. Nitin Kulkarni, Tropical Research Institute, Jabalpur, India

Dr. Robin Wen Jiang Ngiam, National Parks Board, Singapore

Dr. Lional Monod, Natural History Museum of Geneva, Genève, Switzerland.

Dr. Asheesh Shivam, Nehru Gram Bharti University, Allahabad, India

Dr. Rosana Moreira da Rocha, Universidade Federal do Paraná, Curitiba, Brasil

Dr. Kurt R. Arnold, North Dakota State University, Saxony, Germany

Dr. James M. Carpenter, American Museum of Natural History, New York, USA

Dr. David M. Claborn, Missouri State University, Springfield, USA

Dr. Kareen Schnabel, Marine Biologist, Wellington, New Zealand

Dr. Amazonas Chagas Júnior, Universidade Federal de Mato Grosso, Cuiabá, Brasil

Mr. Monsoon Jyoti Gogoi, Assam University, Silchar, Assam, India

Dr. Heo Chong Chin, Universiti Teknologi MARA (UiTM), Selangor, Malaysia

Dr. R.J. Shiel, University of Adelaide, SA 5005, Australia

Dr. Siddharth Kulkarni, The George Washington University, Washington, USA

Dr. Priyadarsanan Dharma Rajan, ATREE, Bengaluru, India

Dr. Phil Alderslade, CSIRO Marine And Atmospheric Research, Hobart, Australia

Dr. John E.N. Veron, Coral Reef Research, Townsville, Australia

Dr. Daniel Whitmore, State Museum of Natural History Stuttgart, Rosenstein, Germany.

Dr. Yu-Feng Hsu, National Taiwan Normal University, Taipei City, Taiwan

Dr. Keith V. Wolfe, Antioch, California, USA

Dr. Siddharth Kulkarni, The Hormiga Lab, The George Washington University, Washington,

D.C., USA

Dr. Tomas Ditrich, Faculty of Education, University of South Bohemia in Ceske

Budejovice, Czech Republic

Dr. Mihaly Foldvari, Natural History Museum, University of Oslo, Norway

Dr. V.P. Uniyal, Wildlife Institute of India, Dehradun, Uttarakhand 248001, India

Dr. John T.D. Caleb, Zoological Survey of India, Kolkata, West Bengal, India

Dr. Priyadarsanan Dharma Rajan, Ashoka Trust for Research in Ecology and the Environment (ATREE), Royal Enclave, Bangalore, Karnataka, India

\section{Fishes}

Dr. Neelesh Dahanukar, IISER, Pune, Maharashtra, India

Dr. Topiltzin Contreras MacBeath, Universidad Autónoma del estado de Morelos, México

Dr. Heok Hee Ng, National University of Singapore, Science Drive, Singapore

Dr. Rajeev Raghavan, St. Albert's College, Kochi, Kerala, India

Dr. Robert D. Sluka, Chiltern Gateway Project, A Rocha UK, Southall, Middlesex, UK

Dr. E. Vivekanandan, Central Marine Fisheries Research Institute, Chennai, India

Dr. Davor Zanella, University of Zagreb, Zagreb, Croatia

Dr. A. Biju Kumar, University of Kerala, Thiruvananthapuram, Kerala, India

Dr. Akhilesh K.V., ICAR-Central Marine Fisheries Research Institute, Mumbai Research

Centre, Mumbai, Maharashtra, India

Dr. J.A. Johnson, Wildlife Institute of India, Dehradun, Uttarakhand, India

\section{Amphibians}

Dr. Sushil K. Dutta, Indian Institute of Science, Bengaluru, Karnataka, India

Dr. Annemarie Ohler, Muséum national d'Histoire naturelle, Paris, France

\section{Reptiles}

Dr. Gernot Vogel, Heidelberg, Germany

Dr. Raju Vyas, Vadodara, Gujarat, India

Dr. Pritpal S. Soorae, Environment Agency, Abu Dubai, UAE.

Prof. Dr. Wayne J. Fuller, Near East University, Mersin, Turkey

Prof. Chandrashekher U. Rivonker, Goa University, Taleigao Plateau, Goa. India

Dr. S.R. Ganesh, Chennai Snake Park, Chennai, Tamil Nadu, India

Dr. Himansu Sekhar Das, Terrestrial \& Marine Biodiversity, Abu Dhabi, UAE
Birds

Dr. Hem Sagar Baral, Charles Sturt University, NSW Australia

Dr. Chris Bowden, Royal Society for the Protection of Birds, Sandy, UK

Dr. Priya Davidar, Pondicherry University, Kalapet, Puducherry, India

Dr. J.W. Duckworth, IUCN SSC, Bath, UK

Dr. Rajah Jayapal, SACON, Coimbatore, Tamil Nadu, India

Dr. Rajiv S. Kalsi, M.L.N. College, Yamuna Nagar, Haryana, India

Dr. V. Santharam, Rishi Valley Education Centre, Chittoor Dt., Andhra Pradesh, India

Dr. S. Balachandran, Bombay Natural History Society, Mumbai, India

Mr. J. Praveen, Bengaluru, India

Dr. C. Srinivasulu, Osmania University, Hyderabad, India

Dr. K.S. Gopi Sundar, International Crane Foundation, Baraboo, USA

Dr. Gombobaatar Sundev, Professor of Ornithology, Ulaanbaatar, Mongolia

Prof. Reuven Yosef, International Birding \& Research Centre, Eilat, Israel

Dr. Taej Mundkur, Wetlands International, Wageningen, The Netherlands

Dr. Carol Inskipp, Bishop Auckland Co., Durham, UK

Dr. Tim Inskipp, Bishop Auckland Co, Durham, UK

Dr. V. Gokula, National College, Tiruchirappalli, Tamil Nadu, India

Dr. Arkady Lelej, Russian Academy of Sciences, Vladivostok, Russia

Dr. Simon Dowell, Science Director, Chester Zoo, UK

Dr. Mário Gabriel Santiago dos Santos, Universidade de Trás-os-Montes e Alto Douro,

Quinta de Prados, Vila Real, Portugal

Dr. Grant Connette, Smithsonian Institution, Royal, VA, USA

Dr. M. Zafar-ul Islam, Prince Saud Al Faisal Wildlife Research Center, Taif, Saudi Arabia

Mammals

Dr. Giovanni Amori, CNR - Institute of Ecosystem Studies, Rome, Italy

Dr. Anwaruddin Chowdhury, Guwahati, India

Dr. David Mallon, Zoological Society of London, UK

Dr. Shomita Mukherjee, SACON, Coimbatore, Tamil Nadu, India

Dr. Angie Appel, Wild Cat Network, Germany

Dr. P.O. Nameer, Kerala Agricultural University, Thrissur, Kerala, India

Dr. Ian Redmond, UNEP Convention on Migratory Species, Lansdown, UK

Dr. Heidi S. Riddle, Riddle's Elephant and Wildlife Sanctuary, Arkansas, USA

Dr. Karin Schwartz, George Mason University, Fairfax, Virginia.

Dr. Lala A.K. Singh, Bhubaneswar, Orissa, India

Dr. Mewa Singh, Mysore University, Mysore, India

Dr. Paul Racey, University of Exeter, Devon, UK

Dr. Honnavalli N. Kumara, SACON, Anaikatty P.O., Coimbatore, Tamil Nadu, India

Dr. Nishith Dharaiya, HNG University, Patan, Gujarat, India

Dr. Spartaco Gippoliti, Socio Onorario Società Italiana per la Storia della Fauna "Giuseppe

Altobello", Rome, Italy

Dr. Justus Joshua, Green Future Foundation, Tiruchirapalli, Tamil Nadu, India

Dr. H. Raghuram, The American College, Madurai, Tamil Nadu, India

Dr. Paul Bates, Harison Institute, Kent, UK

Dr. Jim Sanderson, Small Wild Cat Conservation Foundation, Hartford, USA

Dr. Dan Challender, University of Kent, Canterbury, UK

Dr. David Mallon, Manchester Metropolitan University, Derbyshire, UK

Dr. Brian L. Cypher, California State University-Stanislaus, Bakersfield, CA

Dr. S.S. Talmale, Zoological Survey of India, Pune, Maharashtra, India

Prof. Karan Bahadur Shah, Budhanilakantha Municipality, Kathmandu, Nepal

Dr. Susan Cheyne, Borneo Nature Foundation International, Palangkaraja, Indonesia

Dr. Hemanta Kafley, Wildlife Sciences, Tarleton State University, Texas, USA

\section{Other Disciplines}

Dr. Aniruddha Belsare, Columbia MO 65203, USA (Veterinary)

Dr. Mandar S. Paingankar, University of Pune, Pune, Maharashtra, India (Molecular)

Dr. Jack Tordoff, Critical Ecosystem Partnership Fund, Arlington, USA (Communities)

Dr. Ulrike Streicher, University of Oregon, Eugene, USA (Veterinary)

Dr. Hari Balasubramanian, EcoAdvisors, Nova Scotia, Canada (Communities)

Dr. Rayanna Hellem Santos Bezerra, Universidade Federal de Sergipe, São Cristóvão, Brazil

Dr. Jamie R. Wood, Landcare Research, Canterbury, New Zealand

Dr. Wendy Collinson-Jonker, Endangered Wildlife Trust, Gauteng, South Africa

Dr. Rajeshkumar G. Jani, Anand Agricultural University, Anand, Gujarat, India

Dr. O.N. Tiwari, Senior Scientist, ICAR-Indian Agricultural Research Institute (IARI), New

Delhi, India

Dr. L.D. Singla, Guru Angad Dev Veterinary and Animal Sciences University, Ludhiana, India

Dr. Rupika S. Rajakaruna, University of Peradeniya, Peradeniya, Sri Lanka

Dr. Bahar Baviskar, Wild-CER, Nagpur, Maharashtra 440013, India

Reviewers 2018-2020

Due to pausity of space, the list of reviewers for 2018-2020 is available online.
The opinions expressed by the authors do not reflect the views of the Journal of Threatened Taxa, Wildlife Information Liaison Development Society, Zoo Outreach Organization, or any of the partners. The journal, the publisher, the host, and the partners are not responsible for the accuracy of the political boundaries shown in the maps by the authors.

\footnotetext{
Print copies of the Journal are available at cost. Write to:

The Managing Editor, JoTT,

c/o Wildlife Information Liaison Development Society,

No. 12, Thiruvannamalai Nagar, Saravanampatti - Kalapatti Road,

Saravanampatti, Coimbatore, Tamil Nadu 641035, India

ravi@threatenedtaxa.org
} 


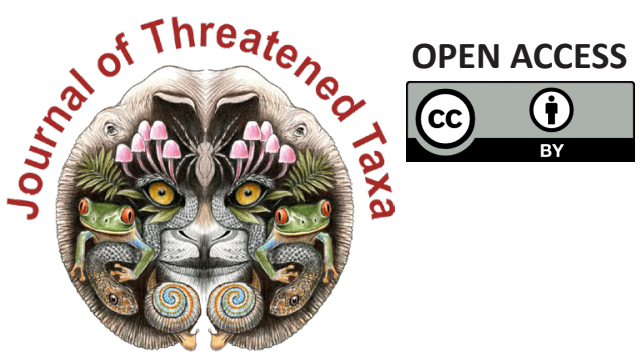

www.threatenedtaxa.org

The Journal of Threatened Taxa (JoTT) is dedicated to building evidence for conservation globally by publishing peer-reviewed articles online every month at a reasonably rapid rate at www.threatenedtaxa.org. All articles published in JoTT are registered under Creative Commons Attribution 4.0 International License unless otherwise mentioned. JoTT allows allows unrestricted use, reproduction, and distribution of articles in any medium by providing adequate credit to the author(s) and the source of publication.

\section{ISSN $0974-7907$ (Online) | ISSN $0974-7893$ (Print)}

\section{September 2021 | Vol. 13 | No. 11 | Pages: 19431-19674 Date of Publication: 26 September 2021 (Online \& Print) DOI: 10.11609/jott.2021.13.11.19431-19674}

Articles

Understanding human-flying fox interactions in the Agusan Marsh Wildlife Sanctuary as basis for conservation policy interventions

- Sherryl L. Paz \& Juan Carlos T. Gonzalez, Pp. 19431-19447

Argentinian odonates (dragonflies and damselflies): current and future distribution and discussion of their conservation

- A. Nava-Bolaños, D.E. Vrech, A.V. Peretti \& A. Córdoba-Aguilar, Pp. 19448-19465

\section{Communications}

The diel activity pattern of small carnivores of Western Ghats, India: a case study at Nelliampathies in Kerala, India

- Devika Sanghamithra \& P.O. Nameer, Pp. 19466-19474

Distribution and threats to Smooth-Coated Otters Lutrogale perspicillata (Mammalia: Carnivora: Mustelidae) in Shuklaphanta National Park, Nepal

- Gopi Krishna Joshi, Rajeev Joshi \& Bishow Poudel, Pp. 19475-19483

Wildlife hunting practices of the Santal and Oraon communities in Rajshahi, Bangladesh - Azizul Islam Barkat, Fahmida Tasnim Liza, Sumaiya Akter, Ashikur Rahman Shome \& M. Fazle Rabbe, Pp. 19484-19491

Ethnozoological use of primates in northeastern India

- Deborah Daolagupu, Nazimur Rahman Talukdar \& Parthankar Choudhury, Pp. 19492-19499

Factors influencing the flush response and flight initiation distance of three owl species in the Andaman Islands

- Shanmugavel Sureshmarimuthu, Santhanakrishnan Babu, Honnavalli Nagaraj Kumara \& Nagaraj Rajeshkumar, Pp. 19500-19508

Birds of Barandabhar Corridor Forest, Chitwan, Nepal

- Saneer Lamichhane, Babu Ram Lamichhane, Kapil Pokharel, Pramod Raj Regmi, Tulasi Prasad Dahal, Santosh Bhattarai, Chiranjibi Prasad Pokheral, Pabitra Gotame,

Trishna Rayamajhi, Ram Chandra Kandel \& Aashish Gurung, Pp. 19509-19526

On some additions to the amphibians of Gunung Inas Forest Reserve, Kedah,

Peninsular Malaysia

- Shahriza Shahrudin, Pp. 19527-19539

Reviews

A review of research on the distribution, ecology, behaviour, and conservation of the Slender Loris Loris lydekkerianus (Mammalia: Primates: Lorisidae) in India

- Mewa Singh, Mridula Singh, Honnavalli N. Kumara, Shanthala Kumar, Smitha D. Gnanaolivu \& Ramamoorthy Sasi, Pp. 19540-19552

Bivalves (Mollusca: Bivalvia) in Malaysian Borneo: status and threats

- Abdulla-Al-Asif, Hadi Hamli, Abu Hena Mustafa Kamal, Mohd Hanafi Idris, Geoffery James Gerusu, Johan Ismail \& Muyassar H. Abualreesh, Pp. 19553-19565

Disentangling earthworm taxonomic stumbling blocks using molecular markers

- Azhar Rashid Lone, Samrendra Singh Thakur, Nalini Tiwari, Olusola B. Sokefun \&

Shweta Yadav, Pp. 19566-19579

A reference of identification keys to plant-parasitic nematodes (Nematoda: Tylenchida) Tylenchomorpha)

- Reza Ghaderi, Manouchehr Hosseinvand \& Ali Eskandari, Pp. 19580-19602

Short Communications

Catalogue of herpetological specimens from Meghalaya, India at the Salim Ali Centre for Ornithology and Natural History

-S.R. Chandramouli, R.S. Naveen, S. Sureshmarimuthu, S. Babu, P.V. Karunakaran \&

Honnavalli N. Kumara, Pp. 19603-19610
A preliminary assessment of odonate diversity along the river Tirthan, Great Himalayan National Park Conservation Area, India with reference to the impact of climate change - Amar Paul Singh, Kritish De, Virendra Prasad Uniyal \& Sambandam Sathyakumar, Pp. 19611-19615

A checklist of orthopteran fauna (Insecta: Orthoptera) with some new records in the cold arid region of Ladakh, India

- M. Ali, M. Kamil Usmani, Hira Naz, Tajamul Hassan Baba \& Mohsin Ali, Pp. 19616-19625

New distribution records of two Begonias to the flora of Bhutan

- Phub Gyeltshen \& Sherab Jamtsho, Pp. 19626-19631

Rediscovery of Aponogeton lakhonensis A. Camus (Aponogetonaceae): a long-lost aquatic plant of India

- Debolina Dey, Shrirang Ramchandra Yadav \& Nilakshee Devi, Pp. 19632-19635

Glyphochloa acuminata (Hack.) Clayton var. laevis (Poaceae): a new variety from central Western Ghats of Karnataka, India

- H.U. Abhijit \& Y.L. Krishnamurthy, Pp. 19636-19639

A cytomorphological investigation of three species of the genus Sonchus L. (Asterales: Asteraceae) from Punjab, India

- M.C. Sidhu \& Rai Singh, Pp. 19640-19644

Dryopteris lunanensis (Dryopteridaceae) - an addition to the pteridophytic diversity of India

- Chhandam Chanda, Christopher Roy Fraser-Jenkins \& Vineet Kumar Rawat, Pp. 1964519648

Notes

First record of Spotted Linsang Prionodon pardicolor (Mammalia: Carnivora:

Prionodontidae) with photographic evidence in Meghalaya, India

- Papori Khatonier \& Adrian Wansaindor Lyngdoh, Pp. 19649-19651

First record of the Eastern Cat Snake Boiga gocool (Gray, 1835) (Squamata: Colubridae) from Tripura, India

- Sumit Nath, Biswajit Singh, Chiranjib Debnath \& Joydeb Majumder, Pp. 19652-19656

First record of the genus Tibetanja (Lepidoptera: Eupterotidae: Janinae) from India - Alka Vaidya \& H. Sankararaman, Pp. 19657-19659

Austroborus cordillerae (Mollusca: Gastropoda) from central Argentina: a rare, little-known land snail

- Sandra Gordillo, Pp. 19660-19662

Intestinal coccidiosis (Apicomplexa: Eimeriidae) in a Himalayan Griffon Vulture Gyps himalayensis

- Vimalraj Padayatchiar Govindan, Parag Madhukar Dhakate \& Ayush Uniyal, Pp. 1966319664

Two new additions to the orchid flora of Assam, India

- Sanswrang Basumatary, Sanjib Baruah \& Lal Ji Singh, Pp. 19665-19670

Wildlife art and illustration - combining black and white ink drawings with colour: some experiments in Auroville, India

- M. Eric Ramanujam \& Joss Brooks, Pp. 19671-19674
Publisher \& Host

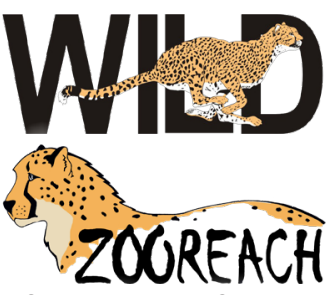

